\title{
Sistemas de administración de estatinas en el tratamiento de la periodontitis: una revisión de literatura
}

\author{
Statins drug delivery systems in the treatment of periodontitis: a literature review
}

Angélica María Cárdenas-Gutiérrez¹, Alejandra Fernández², María Paulina Pieschacón ${ }^{3}$, Gonzalo Navarro ${ }^{4}$

Para citar este artículo: Cárdenas-Gutiérrez AM, Fernández A, Pieschacón MP, Navarro G. Sistemas de administración de estatinas en el tratamiento de la periodontitis: una revisión de literatura. Ustasalud 2018; 17: 40-47

Licencia Creative Commons

\section{(c) (1) (\$) $\ominus$} lo tanto, los lectores pueden acceder libremente a los artículos en su formato .pdf, igualmente podrán descargarlos y difundirlos; sin embargo no podrán modificarlos o alterarlos, adicionalmente se debe reconocer la autoría de las personas que figuran en las publicaciones, pero estas no podrán ser comercializadas.

\begin{abstract}
'Especialista en Periodoncia, Universidad Santo Tomás, Colombia

${ }^{2}$ Especialista en Rehabilitación Oral, Universidad Andrés Bello, Chile
\end{abstract}

${ }^{3}$ Directora Doctorado en Odontología, Universidad Santo Tomás, Colombia

${ }^{4}$ Estudiantes de pregrado, Universidad Andrés Bello, Chile

Autor de Correspondencia:

Cárdenas Gutiérrez Angélica María

Correo electrónico:

angelica.cardenas@ustabuca.edu. co

Recibido para publicación 14 de septiembre 2018 Aceptado para publicación: 1 de noviembre 2018

\section{RESUMEN}

La periodontitis es la inflamación de los tejidos periodontales que puede causar destrucción de los tejidos de soporte del diente, ocasionando pérdida ósea y dental. La periodontitis crónica es causada por bacterias periodontopatógenas como Porphyromonas gingivalis, Aggregatibacter actinomycetemcomitams, Tannerella forsythia y Treponema dentícola, que activan el sistema inmune innato y adaptativo. El tratamiento de la enfermedad periodontal involucra estrategias no quirúrgicas como el raspaje y alisado radicular y tratamiento adjunto que incluye la administración de antibióticos sistémicos y locales como metronidazol, amoxicilina, tetraciclinas, además del uso de estatinas; fármacos utilizados principalmente contra la hiperlipidemia como la simvastatina. El uso de estatinas en la enfermedad periodontal, es dado por su efecto antiinflamatorio, además de estimular la formación ósea, la angiogénesis y su actividad antimicrobiana. Para la aplicación de estos fármacos en el tratamiento de la periodontitis crónica se han utilizado diferentes medios de administración, como: geles, fibras, películas, vesículas, y nanopartículas, destacándose el gel como el sistema preferido para liberar y transportar estatinas, el cual reduce los efectos adversos de la administración sistémica y son más efectivos para la cicatrización ósea. En periodoncia, las estatinas han mostrado una disminución en los parámetros clínicos periodontales después de 6 meses de tratamiento. Sin embargo, existen pocas revisiones temáticas que permitan a la comunidad académica reunir la información pertinente al respecto. Por lo anterior, se vio la necesidad de realizar una revisión de la literatura con el fin de describir los sistemas de administración local y sistémica de estatinas en el tratamiento de la periodontitis.

Palabras clave: Estatinas, periodontitis, sistemas de administración.

\begin{abstract}
Periodontitis corresponds to periodontal tissue inflammation and causing tissue destruction of tooth support leading to bone and tooth loss. Chronic periodontitis is caused by pathogenic bacteria such as Porphyromonas gingivalis, Aggregatibacter actinomycetemcomitans, Tannerella forsythia and Treponema denticola subgingival that activate the innate and adaptative immune system. The treatment of chronic periodontal disease involves non-surgical strategies like scaling and root planning and Adjuncts medical treatment includes the use of systemic and local antibiotics such as metronidazole, amoxicillin, tetracyclines, and statins. Statins are drugs used mainly against hyperlipidemia. Statins most used are simvastatin. In periodontal disease is used to their anti-inflammatory effect. Also statins have positive effects on bone formation, angiogenesis and antimicrobial activity. Different local drug delivery systems have been used in treatment of chronic periodontitis. The systems includes: gels, fibres, films, vesicles, nanoparticles but currently the system prefered to release and transport statins is gel. These drugs are most effective for bone healing. In periodontics, statins has shown decrease of periodontal clinical parameters, after 6 months of treatment. However, there are few thematic reviews that allow the academic community to gather relevant information in this regard. Due to the above, we saw the need to carry out a review of the literature in order to describe the systems of local and systemic administration of statins in the treatment of periodontitis.
\end{abstract}

Keywords: Statins, periodontitis, drug delivery system. 


\section{INTRODUCCIÓN}

La enfermedad periodontal en su forma más severa se encuentra en sexto lugar, dentro de las enfermedades crónicas no trasmisibles, afecta a nivel general el 11,2\% de la población mundial, alrededor de 743 millones de personas. Su prevalencia aumenta con la edad y es más incidente entre los 30 y 40 años ${ }^{1}$. La periodontitis es la inflamación de los tejidos periodontales que puede causar destrucción de los tejidos de soporte del diente, ocasionando pérdida ósea y dental ${ }^{2}$. Esta enfermedad de carácter multifactorial es originada por bacterias periodontopatogénicas, tales como Porphyromonas gingivales, Aggregatibacter actinomycetemcomitans, Tannerella forsythia y Treponema dentícola que activan el sistema inmune innato y adaptativo ${ }^{3}$.

Actualmente se ha determinado un nuevo paradigma en la patogénesis de la enfermedad periodontal, asociada a una disbiosis de la microbiota periodontal que se caracteriza por la sinergia y la potencialización entre entidades bacterianas con funciones distintas que llegan a convertirse en microorganismos patógenos. En otras palabras, se produce alteración en la microbiota ecológicamente equilibrada, la cual está asociada a la homeostasis de los tejidos periodontales ${ }^{4}$.

La patogénesis de la enfermedad periodontal es también asociada a la producción de citoquinas proinflamatorias como la Interleuquina (IL)-1 $\beta$, IL-6 y factor de necrosis tumoral alfa (TNF $\alpha$ ), mediada por los macrófagos y linfocitos $\mathrm{T}$, estas células inducen la reabsorción ósea estimulando la proliferación, activación y diferenciación de los osteoclastos ${ }^{5-7}$. En línea con esto, existe evidencia sobre la asociación enfermedad periodontal y enfermedades sistémicas como ateroesclerosis, artritis reumatoide e infección pulmonar, causada por la diseminación de bacterias, productos bacterianos y moléculas inflamatorias a la circulación sanguínea ocasionando bacteremia e incrementando la inflamación sistémica ${ }^{8-14}$.

A pesar de los avances en la detección de los cambios a nivel molecular en la periodontitis crónica, existen las limitaciones de los marcadores periodontales sistémicos para evaluar la progresión de la enfermedad. Por lo tanto, su diagnóstico está determinado por variaciones en los parámetros clínicos. Los parámetros clínicos evaluados son: profundidad al sondaje (PS), pérdida del nivel de inserción clínico (NIC) y sangrado al sondaje (SS) ${ }^{15}$.
El tratamiento estándar de la periodontitis es el raspaje y alisado radicular que incluye procedimientos no quirúrgicos o quirúrgicos y también estrategias terapéuticas adjuntas como el uso de antibióticos, sistémicos y locales como metronidazol, amoxicilina, azitromicina, tetracliclinas, clorhexidina y también el uso de estatinas ${ }^{16}$.

Las estatinas o inhibidores de la 3-hidroxi-3-metil-glutaril-CoA reductasa (HGM-CoA reductasa) como se conocen farmacológicamente, son medicamentos utilizados generalmente para evitar eventos coronarios cardiacos por su potencialidad para reducir las concentraciones plasmáticas de colesterol y triglicé$\operatorname{ridos}^{17-19}$. Su uso se ha ampliado en los últimos años llegando a implementarse en el área de periodoncia como tratamiento adjunto en la terapia periodontal por sus efectos antinflamatorios, además de su capacidad antioxidante, inmunomoduladorada y angiogénica ${ }^{20,21}$.

En el sistema inmune las estatinas tienen una acción relevante; actúan como inmunosupresoras inhibiendo la presentación antigénica y la activación del complejo mayor de histocompatibilidad (MHC) clase II y a su vez bloqueando la diferenciación de la células tipo ayudadoras o por sus siglas en inglés TH1 (helper type 1), claves en la estimulación a otras células ${ }^{22,23}$.

Dada la falta de revisiones disponibles en español que incluyan los aspectos relevantes sobre la utilización y acción de las estatinas, esta revisión tiene como propósito describir de forma actual los sistemas de administración local y sistémica de las estatinas y sus efectos en el tratamiento de la periodontitis (Tabla 1).

Tabla 1. Sistema de liberación de estatinas

\begin{tabular}{|c|c|}
\hline Estatinas uso sistémico & Estatinas uso local \\
\hline Administración vía oral y enteral & $\begin{array}{l}\text { Administración: in situ durante } \\
\text { el tratamiento de la bolsa perio- } \\
\text { dontal }\end{array}$ \\
\hline $\begin{array}{l}\text { Tipos: Simvastatina, atorvastatina, } \\
\text { lovastatina }\end{array}$ & $\begin{array}{l}\text { Tipos: Simvastatina, atorvas- } \\
\text { tatina }\end{array}$ \\
\hline $\begin{array}{l}\text { Presentación farmacéutica: } \\
\text { Tableta de } 10 \text { y } 20 \mathrm{mg} \text {. }\end{array}$ & $\begin{array}{l}\text { Presentación farmacéutica: } \\
\text { Gel de metilcelulosa de simvas- } \\
\text { tatina de } 0,5 \text { y } 1.2 \mathrm{mg} \\
\text { Gel de atorvastatina al } 1,2 \% \\
\text { Otros: nanopartículas cargadas } \\
\text { con estatinas, vesículas y fibras. }\end{array}$ \\
\hline $\begin{array}{l}\text { Uso: fármaco de doble acción: para } \\
\text { el tratamiento de hiperlipidemia y } \\
\text { enfermedad periodontal. }\end{array}$ & $\begin{array}{l}\text { Uso: tratamiento enfermedad } \\
\text { periodontal. }\end{array}$ \\
\hline
\end{tabular}

RANK L: Ligando del receptor activador para el factor nuclear $\mathrm{kB}$; $\mathrm{mg}$ : miligramos.

Ǘ ITSAASALUD

2018;17:40-41 


\section{Uso sistémico de las estatinas}

La administración enteral $\mathrm{u}$ oral de las estatinas ha mostrado tener un efecto positivo en el tratamiento periodontal realizado en modelo animal y en humanos ${ }^{24,25}$. Hoy en día, las estatinas se pueden prescribir como fármaco de doble acción, tanto en pacientes con hiperlipidemia como en los que padecen enfermedad periodontal. Las moléculas más utilizadas son: la simvastatina (SMV), la atorvastatina y la lovastatina ${ }^{26}$.

El problema de las estatinas a nivel sistémico para el tratamiento de la periodontitis radica en los efectos adversos que pueden producir, relacionados con las dosis administradas sistémicamente y los factores farmacocinéticos. La mayoría de los efectos adversos informados están relacionados con problemas musculoesqueléticos como rabdomiólisis, mialgia y miositis, también trastornos generales, gastrointestinales, endocrinos, alérgicos y nerviosos ${ }^{27}$. A pesar de las nuevas moléculas que se vienen produciendo a fin de mitigar los efectos adversos, estos se siguen presentando, sin embargo, es importante resaltar que aproximadamente el $95 \%$ de los pacientes pueden tolerar el tratamiento con estatinas ${ }^{27}$.

Evidencias de investigaciones en modelo murino y en humanos indican que las estatinas atenúan la inflamación en enfermedad periodontal. Por ejemplo, en animales un estudio demostró que la SMV en tabletas de $20 \mathrm{mg} / \mathrm{kg} /$ día, durante 4 semanas en ratas con síndrome metabólico tuvo un efecto significativo sobre el tejido periodontal, incluida la reducción de la pérdida ósea, reducción del proceso inflamatorio y atenuación de la expresión del ligando del receptor activador para el factor nuclear kB (RANK L) y el factor 2 estimulante de colonias $(\mathrm{CSF} 2)^{24}$.

Los estudios en humanos sobre el efecto de las estatinas administradas de manera sistémica en el tejido periodontal, han demostrado que los pacientes con periodontitis crónica tendrían una ventaja adicional ${ }^{28,29}$. Hay dos explicaciones posibles para esto. En primer lugar, un estado hiperlipidémico acelera un estado proinflamatorio y el uso de estatinas sistémicas podría ayudar a mejorar la inflamación para reducir los niveles de lípidos séricos. En segundo lugar, la acción antiinflamatoria pleiotrópica directa de las estatinas podría ser beneficioso para la salud periodontal ${ }^{29}$.
En línea con lo anterior, Suresh y colaboradores llevaron a cabo un estudio observacional de corte transversal que incluía a 60 pacientes entre 40 y 60 años con diagnóstico de periodontitis crónica generalizada. La mitad de los pacientes estaban medicados con estatinas, con dosis de $20 \mathrm{mg} /$ día durante un período mínimo de 6 meses $^{28}$. La comparación entre los grupos fue basada en el registro de los parámetros clínicos periodontales y los niveles de IL- $1 \beta$ en el fluido gingival crevicular (FGC). Los resultados indicaron que las estatinas tienen un efecto antiinflamatorio sobre la enfermedad periodontal, porque el promedio de los niveles IL-1 $\beta$ en el FGC fue más bajo en pacientes con periodontitis crónica con medicación con estatinas $(180,73 \pm 32,15)$ versus los pacientes con periodontitis crónica sin medicación con estatinas $(308,20 \pm 27,23)^{28}$. Estos efectos positivos podrían estar relacionados con el hecho de que las estatinas bloquean los isoproprenoides, los cuales inhiben la expresión de citoquinas inflamatorias, especialmente IL-1 e IL6, y a su vez regulan el estado hiperlipidémico y atenúan el estado inflamatorio sistémico en general ${ }^{25}$.

En otro estudio, Fentoglu y colaboradores evaluaron el efecto del tratamiento periodontal con terapia adjunta con estatinas en 20 pacientes entre los 34 y 62 años con periodontitis e hiperlipidemia a los 6 meses postratamiento, identificando que los niveles de lípidos séricos (colesterol total y lipoproteínas de alta densidad) y parámetros periodontales como profundidad al sondaje (PS) y sangrado al sondaje (SS) disminuían significativamente entre la medición basal y los 6 meses postratamiento en pacientes hiperlipidémicos medicados con estatinas ${ }^{30}$. Estos resultados demuestran el impacto que tiene el tratamiento con estatinas en la resolución de inflamación sistémica y local a nivel de los tejidos periodontales ${ }^{30}$.

Otros estudios han confirmado que las estatinas administradas sistémicamente pueden tener el beneficio adicional de proteger contra la pérdida dental en pacientes tratados con estatinas comparados con el grupo control ${ }^{25,31}$. En el estudio longitudinal de 5 años liderado por Meisel en el 2014, fueron evaluados 134 pacientes tratados con estatinas donde se demostró una reducción significativa de los niveles séricos de lipoproteínas con menor pérdida dental $(\mathrm{p}=0,03)$, posiblemente asociado a la acción de las 
estatinas sobre el metabolismo óseo y la inhibición de la reabsorción ósea, favoreciendo la arquitectura alveolar y por lo tanto preservando los dientes ${ }^{25}$.

\section{Estatinas en sistemas de liberación local}

En la actualidad, los sistemas de administración local se utilizan para eliminar los efectos sistémicos de la SMV. Este tipo de aplicación se recomienda para el tratamiento de la periodontitis crónica como suplemento en la terapia periodontal, para eliminar cualquier componente inflamatorio residual en el tejido periodontal ${ }^{32}$. Sus diferentes efectos se han demostrado tanto in vitro como in vivo ${ }^{26,33}$.

En general, las estatinas tienen efectos positivos sobre la formación ósea, la cascada inflamatoria, angiogénesis y la actividad antimicrobiana ${ }^{26,32,34}$. Además, la SMV estimula la función osteoblástica aumenta la expresión del factor de crecimiento transformante beta (TGF-B), factor de crecimiento endotelial vascular (VEGF) y proteína morfogenética ósea 2 (BMP2) ${ }^{35,36}$.

Curiosamente, las estatinas se han conjugado con otros fármacos para maximizar sus efectos locales, optimizando la capacidad en la prevención y reducción de la pérdida ósea de periodontitis en modelo animal. La estatina conjugada que fue utilizada en este estudio realizado por Price y colaboradores fue la simvastatina-alendronato-b-ciclodextrina ${ }^{37}$. Los resultados indican que a pesar de que la aplicación de alendronato localmente en ratas con periodontitis inducida experimentalmente, podría tener menor efecto versus el uso sistémico, su acción de inhibición de actividad osteoclástica y antiinflamatorio podría potencializarse al ser combinada con la estatina, obteniendo resultados estadísticamente significativos favoreciendo el mantenimiento del volumen óseo $(\mathrm{p}=0,007)^{37}$.

Por otro lado, se han utilizado diferentes sistemas locales de administración de fármacos en el tratamiento de la periodontitis crónica. Los sistemas incluyen: geles, fibras, películas, vesículas, micropartículas. Sin embargo, de acuerdo con lo revisado actualmente el sistema preferido para liberar y transportar estatinas es el gel ${ }^{32,38,39}$. Por tanto, esta revisión se centra especialmente en esos sistemas de liberación local.

\section{Estatinas liberadas en sistemas de gel}

Gel es un sistema reticulado que presenta dos fases, una continua y otra discontinua. La primera es sólida y la segunda es líquida. El hidrogel tiene una red polimérica y su origen es natural o sintético. Sus propiedades, como la biocompatibilidad y su fácil manejo, han permitido su uso en tejidos locales ${ }^{40}$. Este sistema reticulado también puede incluir células, factores de crecimiento y otros compuestos bioactivos, lo que permite una rápida difusión de los nutrientes hidrofílicos y los metabolitos de las células incorporadas ${ }^{40}$.

Las estatinas en gel usadas en los tejidos periodontales tienen la ventaja de no inducir la respuesta de cuerpo extraño e inflamación, llegando a considerarse como un medio seguro. Además, se puede usar en grandes volúmenes. Sin embargo, pueden presentar el problema de adherirse a los tejidos periodontales ${ }^{33}$.

Los hidrogeles utilizados para el sistema de administración local de estatinas para el tratamiento periodontal son de metilcelulosa, gelatina, ácido hialurónico y poliuretano ${ }^{33,40}$. La más utilizada en esta presentación es la SMV seguida por la atorvastatina, tanto en modelo animal como en humano.

La primera se considera la estatina más potente para la inducción de formación ósea en los defectos creados en la periodontitis crónica ${ }^{38,40,41}$. Los cambios encontrados a nivel óseo son: aumento en el espesor de la cresta alveolar con una disminución en los defectos de pérdida ósea interproximal, en modelo animal tratados con simvastatina de $0,5 \mathrm{mg}^{33}$. Los resultados demostraron que el espesor del reborde edéntulo era $29 \%$ más grande en los modelos tratados con simvastatina comparados con los controles (gel sin estatina) y en relación con el material, al disminuir la viscosidad del gel de metilcelulosa se logró una mejor retención y aplicación dentro de los defectos óseos asegurando la formación de nuevo tejido óseo ${ }^{33}$.

Otra investigación sobre este tema fue hecha por Vaziri y col., quienes demostraron que la administración local de simvastatina en el tejido subperióstico redujo el grado de resorción ósea y la pérdida de inserción, en una condición de periodontitis inducida experimentalmente en ratas ovariectomizadas. Sugi- 
rieron que las estatinas de administración local pueden proteger las condiciones inflamatorias clínicas ${ }^{42}$.

Más recientemente, se ha considerado que la atorvastatina al 1,2\% en gel de metilcelulosa tiene un efecto significativo en individuos con periodontitis crónica. Clínicamente se ha demostrado que la atorvastatina como fármaco de administración local mejora los parámetros periodontales después del tratamiento periodontal tradicional. Del mismo modo, mejora radiográficamente en zonas con defectos óseos ${ }^{38}$.

Los estudios en humanos sobre los efectos de las estatinas en los tejidos periodontales en pacientes sanos o con enfermedades sistémicas sugirieron también un efecto antiinflamatorio ${ }^{43}$. Pradeep y col. examinaron el efecto tópico del gel de metilcelulosa de SMV al 1,2\%, inyectado en las bolsas periodontales de 60 individuos con periodontitis crónica. Este estudio ilustró la capacidad del SMV de uso tópico en el mejoramiento de los parámetros clínicos y radiográficos. Cambios en la reducción de parámetros clínicos como el SS $(2,32 \pm 0,80$ versus $0,50 \pm 0,68)$ y PS $(1,20 \pm 1,24 \mathrm{~mm}$ versus $4,26 \pm 1,59 \mathrm{~mm}$ ), más la ganancia de nivel de inserción clínico $(1,63 \pm 1,99 \mathrm{~mm}$ versus $4,36 \pm 1,92 \mathrm{~mm})$ después de 6 meses postratamiento fueron evidentes comparado con el grupo control ${ }^{38}$.

Con respecto a la acción de estatinas locales en pacientes con afectación sistémica o fumadores, la inyección de SMV metilcelulosa al 1,2\% en las bolsas periodontales de fumadores e individuos con diabetes tipo 2, llevó a cambios notables en los parámetros clínicos después de 9 meses de tratamiento como terapia adjunta $^{41}$.

En cultivos celulares, la atorvastatina puede regular la producción de RANKL y osteoprotegerina, moléculas que en condiciones homeostáticas limitan la osteoclastogénesis y resorción ósea. La atorvastatina también inhibe la liberación de metaloproteinasas de matriz (MMP), MMP1 y MMP- $3^{44,45}$. Es importante tener en cuenta que el colágeno tipo I es el principal componente del periodonto y es degradado por colagenasas como la MMP-1, involucrada en el inicio de la degradación del mismo ${ }^{46}$. A pesar de los diferentes usos y resultados beneficiosos del gel de estatina en el tratamiento periodontal, se ha demostrado que no permiten la liberación homogénea y continúa de las estatinas $^{33}$.

\section{Sistema de nanopartículas de estatina}

A pesar de que desde el año 2010 se han sintetizado nanopartículas cargadas con estatinas, estas solo se han utilizado para prevención y tratamiento de arterioesclerosis y tratamiento de hipertensión arterial pulmonar y el cáncer ${ }^{47-49}$. El uso de la estatina en tamaño nanométrico, provee control de los eventos adversos en comparación con las dosis sistémicas, gracias a su aplicación selectiva y específica. En su mayoría están compuestos de copolímeros polilácticos altamente biocompatibles ${ }^{50}$.

Estudios en ratas han demostrado que la aplicación de nanoesferas de estatina promueve la cicatrización ósea y puede ser terapéuticamente útil en humanos si se aplican directamente en el área de interes ${ }^{51}$. Es importante tener en cuenta que hay una gran falta de estudios que evalúen el uso de estatinas con nanotecnología para su aplicación en humanos.

\section{CONCLUSIÓN}

El uso de las estatinas en periodoncia como tratamiento adjunto en la terapia periodontal puede llegar a ser beneficioso por sus efectos antiinflamatorios, además de su capacidad antioxidante, entre otros.

Las estatinas aplicadas de manera tópica podrían contrarrestar la inflamación y conducir la regeneración de tejidos blandos y duros en el tratamiento de la enfermedad periodontal, sin los efectos adversos de la administración oral. Por lo tanto, las estatinas en gel, son la mejor opción en especial simvastatina un gel de metilcelulosa al 1,2\%, seguida por la atorvastatina. A pesar de los resultados beneficiosos, se ha demostrado que el gel no permite la liberación homogénea y continúa sobre los tejidos periodontales.

Finalmente, el reto de las estatinas en gel radica en reducir la inflamación y producir regeneración tisular con un medicamento estable y biocompatible; idealmente con una liberación controlada, evitando los efectos secundarios indeseables que se producen con la administración sistémica del medicamento. 


\section{REFERENCIAS}

1. Tonetti M, Jepsen S, Jin L, Otomo-Corgel J. Impact of the global burden of periodontal diseases on health, nutrition and wellbeing of mankind: A call for global action. J Clin Periodontol. 2017;44(5):456-462. DOI: 10.1111/ jcpe.12732.

2. Hajishengallis G. Periodontitis: from microbial immune subversion to systemic inflammation. Nat Rev Immunol. 2015;15(1):30-44. DOI: 10.1038/nri3785.

3. Garlet GP. Destructive and protective roles of cytokines in periodontitis: a re-appraisal from host defense and tissue destruction viewpoints. J Dent Res. 2010;89(12):1349-63. DOI: $10.1177 / 0022034510376402$.

4. Hajishengallis G. Korostoff J. Revisiting the Page \& Schroeder model: the good, the bad and the unknowns in the periodontal host response 40 years later. Periodontol 2000. 2017;75(1):116-151. DOI: 10.1111/prd.12181.

5. Chen XT, Chen LL, Tan JY, Shi DH, Ke T, Lei LH. Th17 and Th1 Lymphocytes Are Correlated with Chronic Periodontitis. Immunol Invest. 2016;45(3):243-54. DOI: 10.3109/08820139.2016.1138967.

6. Estanislau IM, Terceiro IR, Lisboa MR, Teles P, Carvalho R, Martins R, Moreira M. Effects of statins on the treatment of chronic periodontitis - a systematic review. J Clin Pharmacol. 2015;79(6):877-885. DOI: 10.1111/ bcp. 12564 .

7. Gmiterek A, Kłopot A, Wójtowicz H, Trindade SC, Olczak M, Olczak T. Immune response of macrophages induced by Porphyromonas gingivalis requires $\mathrm{HmuY}$ protein. Immunobiology. 2016;221(12):1382-1394. DOI: 10.1016/j.imbio.

8. Trombelli L, Farina R, Silva C, Tatakis DN. Plaque-induced gingivitis: case definition and diagnostic considerations. J Clin Periodontol. 2018;89:S46-S73. DOI: 10.1002/ JPER.17-0576.

9. Ridker PM, Silvertown JD. Inflammation, C-reactive protein, and atherothrombosis. J Periodontol. 2008;79(8 Suppl):1544-1551. DOI: 10.1902/jop.2008.080249.

10. Pearson TA, Mensah GA, Alexander RW, Anderson JL, Cannon RO. Markers of inflammation and cardiovascular disease: application to clinical and public health practice: A statement for healthcare professionals from the Centers for Disease Control and Prevention and the American Heart Association. Circulation. 2003;107(3):499-511.
11. Chee B, Park B, Bartold M. Periodontitis and type II diabetes: a two way relationship. Int J Based Healthc. 2013;11(4):317-29. DOI: 10.1111/1744-1609.12038.

12. García R, Henshaw M, Krall E. Relationship between Periodontal disease and systemic health. Periodontol 2000. 2001;25:21-36.

13. Otomo-Corgel J, Pucher JJ, Rethman MP, Reynolds MA. State of the science: chronic periodontitis and systemic health. J Evid Based Dent Pract. 2012;12(3 Suppl):20-8. DOI: $10.1016 /$ S1532-3382(12)70006-4.

14. Azarpazhooh A, Tenenbaum HC. Separating fact from fiction: use of high-level evidence from research syntheses to identify diseases and disorders associated with periodontal disease. J Can Dent Assoc. 2012;78:c25.

15. Górska R, Gregorek H, Kowalski J, Laskus-Perendyk A, Syczewska M, Madaliński K. Relationship between clinical parameters and cytokine profiles in inflamed gingival tissue and serum samples from patients with chronic periodontitis. J Clin Periodontol. 2003;30(12):104652. DOI: $10.1046 / \mathrm{j} .0303-6979.2003 .00425 . x$.

16. Smiley CJ, Tracy SL, Abt E, Michalowicz BS, John MT, Gunsolley J, Cobb CM. Evidence-based clinical practice guideline on the nonsurgical treatment of chronic periodontitis by means of scaling and root planing with or without adjuncts. J Am Dent Assoc. 2015;146(7):525-35. DOI: 10.1016/j.adaj.2015.01.026.

17. Tvaryanas A, Wagner J, Maupin G. Statins and musculoskeletal conditions in U.S. Air Force Active Duty Aviators. Aerosp Med Hum Perform. 2018;89(5):421-427. DOI: 10.7205/MILMED-D-17-00058.

18. Gunjiganur VG, Emani S, Mehta DS, Bhandari S. Effect of $1.2 \%$ of simvastatin gel as a local drug delivery system on gingival crevicular fluid interleukin- 6 and interleukin-8 levels in non-surgical treatment of chronic periodontitis patients. Archives of Oral Biology. 2017;82:55-61. DOI: 10.1016/j.archoralbio.2017.05.022.

19. Stancu C, Sima A. Statins: mechanism of action and effects. J Cell Mol Med. 2001;5(4):378-387.

20. Cicek Ari V, Ilarslan YD, Erman B, Sarkarati B, Tezcan I, Karabulut E. Statins and IL-1 $\beta$, IL-10, and MPO Levels in Gingival Crevicular Fluid: Preliminary Results. Inflammation. 2016;39(4):1547-57. DOI: 10.1007/ s10753-016-0390-7.

21. Shah M, Muley P, Muley A. Are statins worthy for treatment of periodontitis? A systematic review and meta-analysis. Adv Hum Biol. 2017;7:8-14. 
22. Chen L, Nakano K, Kimura S, Matoba T, Iwata E, Miyagawa M, et al. Nanoparticle-mediated delivery of pitavastatin into lungs ameliorates the development and induces regression of monocrotaline-induced pulmonary artery hypertension. Hypertension. 2011;57(2):343-50. DOI: 10.1161/HYPERTENSIONAHA.110.157032.

23. Kim YC, Kim KK, Shevach EM. Simvastatin induces Foxp3+ $\mathrm{T}$ regulatory cells by modulation of transforming growth factor-beta signal transduction. Immunology. 2010; 130(4):484-93. DOI: 10.1111/j.1365-2567.2010.03269.x.

24. Jin J, Machado E, Yu H, Zhang X, Lu Z, Li Y, Lopes-Virella $M$, et al. Simvastatin Inhibits LPS-induced Alveolar Bone Loss during Metabolic Syndrome. J Dent Res 2014;93(3):294-9. DOI: 10.1177/0022034513516980.

25. Meisel P, Kroemer HK, Nauck M, Holtfreter B, Kocher T. Tooth loss, periodontitis, and statins in a population-based follow-up study. J Periodontol. 2014;85(6):e160-8. DOI: 10.1902/jop.2013.130456.

26. Emani S, Gunjiganur GV, Mehta DS. Determination of the antibacterial activity of simvastatin against periodontal pathogens, Porphyromonas gingivalis and Aggregatibacter actinomycetemcomitans: An in vitro study. Contemp Clin Dent 2014;5(3):377-82. DOI: 10.4103/0976-237X.137959.

27. Thompson P, Clarkson P, Karas RH. Statin-Associated Myopathy. JAMA. 2003;289(13):1681-90.

28. Suresh S, Narayana S, ayakumar P, Sudhakar U, Pramod V. Evaluation of anti-inflammatory effect of statins in chronic periodontitis. Indian J Pharmacol. 2013; 45(4):391-4. DOI: 10.4103/0253-7613.115017.

29. Sangwan A, Tewari S, Singh H, Sharma RK, Narula SC Periodontal status and hyperlipidemia: statin users versus non-users. J Periodontol. 2013;84(1):3-12. DOI: 10.1902/ jop.2012.110756.

30. Fentoğlu O, Sözen T, Oz SG, Kale B, Sönmez Y, Tonguç MO, et al. Short-term effects of periodontal therapy as an adjunct to anti-lipemic treatment. Oral Dis. 2010;16(7):64854. DOI: 10.1111/j.1601-0825.2010.01668.x.

31. Cunha-Cruz J, Saver B, Maupome G, Hujoel PP. Statin use and tooth loss in chronic periodontitis patients. J Periodontol. 2006;77(6):1061-6.

32. Elavarasu S, Suthanthiran TK, Naveen D. Statins: A new era in local drug delivery. Journal of Pharmacy \& Bioallied Sciences. 2012;4(Suppl 2):S248-51. DOI: 10.4103/0975-7406.100225.
33. Morris M, Lee Y, Lavin, M, Giannini P, Schmid, M., Marx $\mathrm{D}$, et al. Injectable simvastatin in periodontal defects and alveolar ridges: pilot studies. Journal of Periodontology. 2008;79(8):1465-73. DOI: 10.1902/jop.2008.070659.

34. Fajardo ME, Rocha ML, Sánchez-Marin FJ, Espinosa-Chávez EJ. Effect of atorvastatin on chronic periodontitis: a randomized pilot study. J Clin Periodontol 2010;37(11):101622. DOI: 10.1111/j.1600-051X.2010.01619.x.

35. Seto H, Ohba H, Tokunaga K., Hama H., Horibe M, Nagata, T. Topical administration of simvastatin recovers alveolar bone loss in rats. J Periodontal Res. 2008;43(3):2617. DOI: 10.1111/j.1600-0765.2007.01024.x.

36. Mundy G, Garrett R, Harris S, Chan J, Chen D, Rossini $\mathrm{G}$, et al. Stimulation of bone formation in vitro and in rodents by statins. Science. 1999;286(5446):1946-9.

37. Price U, Le HO, Powell SE, Schmid MJ, Marx DB, Zhang $\mathrm{Y}$, et al. Effects of local simvastatin-alendronate conjugate in preventing periodontitis bone loss. J Periodontal Res. 2013;48(5):541-8. DOI: 10.1111/jre.12036.

38. Pradeep AR, Thorat MS. Clinical effect of subgingivally delivered simvastatin in the treatment of patients with chronic periodontitis: a randomized clinical trial. J Periodontol. 2010;81(2):214-22. DOI: 10.1902/ jop.2009.090429.

39. Garg S, Pradeep AR. 1.2\% Rosuvastatin and 1.2\% Atorvastatin Gel Local Drug Delivery and Redelivery in the Treatment of Class II Furcation Defects: A Randomized Controlled Clinical Trial. J Periodontol. 2017;88(3):259265. DOI: 10.1902/jop.2016.160399.

40. Oryan A, Kamali A, Mosh A. Potential mechanisms and applications of statins on osteogenesis: Current modalities, conflicts and future directions. J Control Release. 2015;215:12-24. DOI: 10.1016/j.jconrel.2015.07.022.

41. Rao N, Pradeep A, Bajaj P, Kumari M, Naik S. Simvastatin local drug delivery in smokers with chronic periodontitis: a randomized controlled clinical trial. Aus Dent J. 2013;58 (2):156-62. DOI: 10.1111/adj.12042.

42. Vaziri H, Naserhojjati-Roodsari R, Tahsili-Fahadan N, Khojasteh A, Mashhadi-Abbas F, Eslami B, et al. Effect of simvastatin administration on periodontitis-associated bone loss in ovariectomized rats. J Periodontol. 2007;78(8):1561-7.

43. Stein S, Dean IN, Rawal SY, Tipton DA. Statins regulate interleukin-1 $\beta$-induced RANKL and osteoprotegerin production by human gingival fibroblasts. J Periodontal Res. 2011;46(4):483-90. DOI: 10.1111/j.1600-0765.2011.01364.x. 
44. Grover H, Luthra S, Maroo S, Maroo N. The pleotropic role of statins: Could it be the imminent host modulation agent in periodontics? Dent Res J (Isfahan). 2013;10(2):143-8.

45. Kamio K, Liu X, Sugiura H, Togo S, Kawasaki S, Wang X, et al. Statins inhibit matrix metalloproteinase release from human lung fibroblasts. Eur Respir J. 2010;35(3):637-46. DOI: 10.1183/09031936.00134707.

46. Gursoy UK, Könönen E, Huumonen S, Tervahartiala T, Pussinen PJ, Suominen AL, et al. Salivary type I collagen degradation end-products and related matrixmetalloproteinases in periodontitis. J Clin Periodontol 2013;40(1):18-25. DOI: 10.1111/jcpe.12020.

47. Koga J, Matoba T, Egashira K. Anti-inflammatory Nanoparticle for Prevention of Atherosclerotic Vascular Diseases. J Atheroscler Thromb. 2016;23(7):757-65. DOI: $10.5551 /$ jat.35113.
48. Wu Y, Wang Z, Liu G, Zeng X, Wang X, Gao Y, et al. Novel Simvastatin-Loaded Nanoparticles Based on Cholic Acid-Core Star-Shaped PLGA for Breast Cancer Treatment. J Biomed Nanotechnol. 2015;11(7):1247-60.

49. Kretzer IF, Maria DA, Guido MC, Contente TC, Maranhão RC. Simvastatin increases the antineoplastic actions of paclitaxel carried in lipid nanoemulsions in melanoma-bearing mice. Int J Nanomedicine. 2016;11:885-904. DOI: $10.2147 /$ IJN.S88546.

50. Tiwari R, Pathak K. Statins therapy: a review on conventional and novel formulation approaches. J Pharm Pharmacol. 2011;63(8):983-98. DOI: 10.1111/j.2042-7158.2011.01273.x.

51. Garrett IR, Gutierrez GE, Rossini G, Nyman J, McCluskey B, Flores A, et al. Locally delivered lovastatin nanoparticles enhance fracture healing in rats. J Orthop Res. 2007;25(10):1351-7. 